\title{
Myopic deconvolution method for adaptive optics images of stellar fields
}

\author{
T. Fusco ${ }^{1,2}$, J.-P. Véran ${ }^{3}$, J.-M. Conan ${ }^{1}$, and L.M. Mugnier ${ }^{1}$ \\ 1 ONERA, BP. 72, 92322 Chatillon Cedex, France \\ 2 Département d'Astrophysique, URA 709 du CNRS, UFR Faculté des Sciences, Université de Nice-Sophia Antipolis, Parc \\ Valrose, 06034 Nice Cedex, France \\ 3 Herzberg Institute of Astrophysics, Dominion Astrophysical Observatory, 5071 west Saanich Road, Victoria, B.C. V8X 4M6, \\ Canada
}

Received April 22; accepted July 23, 1998

\begin{abstract}
The atmospheric turbulence severely limits the angular resolution of ground based telescopes. When using Adaptive Optics compensation, the wavefront sensor data permit the estimation of the residual PSF. Yet, this estimation is imperfect. A "myopic" deconvolution method which takes into account this estimation and its associated noise is presented. This technique is applied to adaptive optics corrected images of star fields. It is based on a stochastic approach which uses the available information about the object and about the PSF. This technique allows the simultaneous restoration of star parameters (position with sub-pixel precision and accurate photometry) and of the PSF. The algorithm is successfully applied both to simulated and to experimental data.
\end{abstract}

Key words: techniques: adaptive optics - methods: image processing

\section{Introduction}

Atmospheric turbulence severely limits the angular resolution of ground based telescopes. Many techniques have been proposed to reach the diffraction limit of large optical instruments. A. Labeyrie (Labeyrie 1970) suggested to record series of short exposure images to freeze the turbulence effects. Various numerical post-processing methods (Knox \& Thompson 1974), (Weugekt 1977) then permit the reconstruction of the observed object.

Send offprint requests to: T. Fusco

Correspondence to: authors:

Phone: 33 (0)1 467340 40; fax: 33 (0)1 46734171 .

E-mail: $\{\mathrm{NAME}\} @$ onera.fr

URL: http://www.onera.fr/activites/iohr/en
As an alternative, Adaptive Optics [AO] (Babcock 1953; Rousset et al. 1990) is a real-time technique which compensates for the random variation of the turbulent wave front. This compensation enables the recording of high spatial resolution long exposure images. However, even if the object's spatial frequencies are preserved up to the diffraction limit of the telescope, they are often severely attenuated since the AO correction is only partial. It is therefore necessary to use image processing techniques to improve the quality of the recovered object (Thiebaut \& Conan 1995; Christou et al. 1997).

We consider here stellar fields. The main problem with images of such objects is that the AO partial correction induces a halo around each star. When two stars are too close, their halos overlap, and photometric and astrometric measurements are difficult. Different post-processing algorithms have been proposed to deal with this problem (CLEAN (Hogbom 1974), DAOPHOT (Stetson 1987), PLucy algorithm (Lucy 1994), (Hook \& Lucy 1994)) but they all require that the PSF be pre-determined accurately. In certain cases, especially with a short exposure time, very partial AO correction or uncalibrated aberrations, the determination of the PSF is too rough to yield good astrometric and photometric results.

In this paper, we propose a deconvolution method for such star field observations. This deconvolution technique permits the restoration of both the object and the PSF. We call it a "myopic" deconvolution, because it incorporates the knowledge of the Point Spread Function [PSF] structure and variability (as opposed to "blind" deconvolution where the PSF is assumed to be completely unknown). The strong knowledge of the object (point-like object) is also used, which allows a sub-pixel and a good photometric restoration.

In section two, an overview of the formation of $\mathrm{AO}$ corrected images is presented. In section three, the myopic 
deconvolution is introduced. In section four, the method used to estimate the average and the variability of the PSF is detailed. This method is based on the processing of the wavefront sensor data recorded simultaneously with the AO image. In section five simulation results are presented. They show that our method is able to accurately recover the object parameters (astrometry and photometry) and the PSF even in unfavorable cases (low flux, very partial AO correction).

In the last section experimental results acquired with the Canada-France-Hawaii Telescope adaptive optics system are presented. The myopic deconvolution allows a good restoration despite the static aberrations present in the true PSF but not measured by the wavefront sensor.

\section{Image formation in adaptive optics}

Within the isoplanatic patch, the AO corrected image is a convolution of the true object $(o(\mathbf{r}))$ with a residual PSF $(h(\mathbf{r}))$ characterizing the turbulence, the telescope and the AO bench. The detected intensity distribution $d(\mathbf{r})$ of the corrected image is given by:

$d(\mathbf{r})=o(\mathbf{r}) \star h(\mathbf{r})+n(\mathbf{r})$

$n(\mathbf{r})$ is an additive zero mean random process that accounts for the noise affecting the image (photon and detector noise). The AO correction is always partial and does not provide a full compensation for the atmospheric turbulence. The correction performance depends on the observing conditions: turbulence strength characterized by the Fried parameter $\left(r_{0}\right)$ (Fried 1965), magnitude and angular size of the source used for wavefront sensing. It also depends on the AO system itself: number of corrected modes, servo-loop bandwidth etc. (Rousset et al. 1990; Rigaut 1991; Conan 1994). The partially corrected image can be fully characterized by the PSF $h(\mathbf{r})$.

A posteriori deconvolution is therefore required to estimate the object $o(\mathbf{r})$ from the data $d(\mathbf{r})$.

\section{The myopic deconvolution method}

It is well-known that image restoration is an ill-posed problem which needs to be regularized through the use of additional a priori information (Tikhonov \& Arsenin 1977), and (Demoment 1989) for a review.

The goal of myopic deconvolution is to restore both the object and the PSF knowing the image intensity measurements and the a priori information.

We model our observed object (field of stars) by a sum of Dirac functions (Gunsay \& Jeffs 1995). Its intensity is then given by:

$o(\boldsymbol{r})=\sum_{i}^{n} \alpha_{i} \delta\left(\boldsymbol{r}-\boldsymbol{a}_{i}\right)$

where $\boldsymbol{a}_{i}$ and $\alpha_{i}$ are the position vector and the intensity of the $i$ th star respectively and are the object parameters we are seeking. We consider that $n$, the number of stars, is a known parameter.

A constant or slowly variable parameter can also be added to account for sky background and underlying emission (Lucy 1994).

The deconvolution method we use is based on a stochastic approach i.e., we consider object parameters and PSF as outcomes of stochastic processes (Conan 1998).

Bayes' rule combines the likelihood of the data with the a priori distribution of the parameters (object and PSF, which are decorraleted processes) into the a posteriori probability distribution of the unknowns $\left(\boldsymbol{a}_{i}, \alpha_{i}\right.$ and $h)$ :

$p\left(\boldsymbol{a}_{i}, \alpha_{i}, h \mid d\right) \propto p\left(d \mid \boldsymbol{a}_{i}, \alpha_{i}, h\right) \times p\left(\boldsymbol{a}_{i}, \alpha_{i}\right) \times p(h)$

where $p(A \mid B)$ means conditional probability of $A$ given $B$. $p\left(d \mid \boldsymbol{a}_{i}, \alpha_{i}, h\right)$ corresponds to the noise probability law (likelihood term) and accounts for the noise affecting the image: photon and detector noise. In a first approximation we choose a stationary white Gaussian statistics for the noise probability, although a non-stationary gaussian or poisson plus gaussian statistics would be more accurate.

$p\left(\boldsymbol{a}_{i}, \alpha_{i}\right)$ reflects the a priori knowledge (or prior) on the object parameters. In addition to the reparametrization itself, which contains a strong constraint on the object, we also use:

- the positivity of the star intensities, enforced in our case by a reparametrization of intensities by magnitudes. $\alpha_{i}=10^{-0.4 * m_{i}}$ where $m_{i}$ is the star magnitude. - an upper limit on the star intensities

$\left(\sum_{i} \alpha_{i}<\right.$ whole image flux $)$.

Similarly, $p(h)$ reflects the a priori knowledge on the PSF. It can be reasonably assumed to be Gaussian and is therefore characterized by its first and second moments (Conan et al. 1998): a mean PSF $\left(h_{\mathrm{m}}\right)$ and a Power Spectral Density [PSD] $\left(P S D_{\mathrm{h}}\right)$. This PSD can be viewed as a per frequency variance of the Optical Transfer Function [OTF] (Fourier Transform of the PSF) around its mean value.

The method used to calculate $h_{\mathrm{m}}$ and $P S D_{\mathrm{h}}$ using the Wave-front sensor (WFS) data or the image of a reference star will be described in Sect. 4 .

Following the Maximum A Posteriori (MAP) approach, a set of estimated parameters is given by the maximization of $p\left(\boldsymbol{a}_{i}, \alpha_{i}, h \mid d\right)$, the a posteriori probability law:

$\left[\hat{\alpha}_{i}, \hat{\boldsymbol{a}}_{i}, \hat{h}\right]_{\text {map }}=\operatorname{argmax}_{\alpha_{i}, \boldsymbol{a}_{i}, h}\left[p\left(\boldsymbol{a}_{i}, \alpha_{i}, h \mid d\right)\right]$.

In this case, maximizing the probability corresponds to minimizing the following criterion $\left(-\log p\left(\boldsymbol{a}_{i}, \alpha_{i}, h \mid d\right)\right)$ in the Fourier domain:

$J\left(\alpha_{i}, \boldsymbol{a}_{i}, \tilde{h}(\boldsymbol{f})\right)=\sum_{\boldsymbol{f}=0}^{N^{2}} \frac{1}{\sigma^{2}}\left|\alpha_{i} \exp \left(\frac{-2 j \pi}{N} \boldsymbol{f} \boldsymbol{a}_{i}\right) \times \tilde{h}(\boldsymbol{f})-\tilde{d}(\boldsymbol{f})\right|^{2}$ 


$$
+\sum_{\boldsymbol{f}=0}^{N^{2}} \frac{\left|\tilde{h}(\boldsymbol{f})-\tilde{h}_{\mathrm{m}}(\boldsymbol{f})\right|^{2}}{P S D_{\mathrm{h}}(\boldsymbol{f})}
$$

where . denotes a Fourier transform, $N^{2}$ the pixel number, $\sigma^{2}$ the noise variance and $\boldsymbol{f}$ the spatial frequency.

The first term of Eq. (5), is the likelihood term which takes into account the object reparametrisation.

The second term is a regularization term on the PSF. It draws the actual OTF towards its mean value $\tilde{h}_{\mathrm{m}}$ with a stiffness related to its PSD. This term avoids a noise propagation and amplification on the PSF (cf. Sect. 5) as well as the trivial solution (PSF $=$ image and all the sources $=0$ except one delta function).

This leads to a strong decrease of the number of parameters. In the case of a $128 \times 128$ pixels image of a binary star, the number of parameters drops from 16384 (if the object parameters are the intensity on each pixel) to 6 (two intensities, two position vectors). It therefore allows a better precision on these parameters; in particular, a sub-pixel determination of star position - meaning a fraction of $\frac{\lambda}{2 D}$ for Shannon sampled images - and an accurate photometry measurement.

Also, fewer parameters to estimate lead to a faster convergence.

We now turn to the different ways of estimating the PSF and its associated PSD.

\section{Mean PSF and PSD estimation}

The correction achieved by adaptive optics depends on the magnitude and angular size of the reference object, as well as on the strength of atmospheric turbulence. As a result, the PSF evolves both in time and space. Each image has a different PSF, which is difficult to determine.

One possibility to estimate the PSF, is to image a star near the observed object (Northcott 1996). This technique however presents some major problems: waste of observation time, small sky coverage, evolution of the turbulence between object and reference star acquisition.

Recently, a method has been proposed to accurately retrieve the mean PSF $\left(h_{\mathrm{m}}\right)$ from the data accumulated by the AO control system during the acquisition (Veran et al. 1997). This mean PSF however corresponds to an image with an infinite integration time. $h_{\mathrm{m}}$ is obtained using the theoretical expression:

$\tilde{h}_{\mathrm{m}}(\boldsymbol{f})=\exp \left(-\frac{1}{2} D_{\phi_{\mathrm{r}}(\lambda \boldsymbol{f})}\right) \times O T F_{\text {free }}(\boldsymbol{f})$

where $D_{\phi_{\mathrm{r}}}(\lambda \boldsymbol{f})=<\left[\phi_{\mathrm{r}}(\boldsymbol{\rho})-\phi_{\mathrm{r}}(\boldsymbol{\rho}+\lambda \boldsymbol{f})\right]^{2}>$ is the residual phase structure function estimated from the AO data and $O T F_{\text {free }}$ is the aberration-free OTF. Note that, in practice, the WFS data never allow the calculation of the true $\phi_{\mathrm{r}}$ but give a noisy measurement of this phase (Veran et al. 1997).

$h_{\mathrm{m}}$ differs from the actual finite time PSF because of the so-called turbulence noise, related to the residual speckles in the AO corrected images. The shorter the integration time, the larger the turbulence noise. The variance of this noise on the optical transfer function for an integration time $T_{\mathrm{i}}$ can be estimated at each spatial frequency by (Conan 1994):

$\sigma_{\text {turb }}^{2}(\boldsymbol{f})=\frac{\tau_{\mathrm{s}}}{T_{\mathrm{i}}}\left(S T F(\boldsymbol{f})-\left|\tilde{h}_{\mathrm{m}}(\boldsymbol{f})\right|^{2}\right)$

where $S T F(\boldsymbol{f})$ is the Speckle Transfer Function (Roddier 1981) and $\tau_{\mathrm{s}}$ is the coherence time characterizing the turbulence evolution. Using some approximations, the STF can be expressed as a function of $\tilde{h}_{\mathrm{m}}$ (Conan 1994) and can therefore be also estimated from the AO loop data.

The difficulty lies in the estimation of the coherence time $\tau_{\mathrm{s}}$. It corresponds roughly to the turbulence evolution time. Without $\mathrm{AO}$ correction, $\tau_{\mathrm{s}}$ is the so-called speckle boiling time characterizing the speckle life time. An approximate value is given by Roddier (Roddier et al. 1982). It could be different in the case of AO correction and certainly depends on the correction degree. However we assume, in a first approximation, that the expression given by Roddier is valid here:

$\tau_{\mathrm{s}}=0.36 \frac{r_{0}}{\bar{v}}$

where $r_{0}$ is the Fried parameter of the turbulence and $\bar{v}$ is the mean wind speed across the different turbulence layers. $r_{0}$ can be computed from the AO loop data (Veran et al. 1997) as well as $\bar{v}$ (Conan et al. 1995).

With accurate models of the servo-loops, WFS, deformable mirror, a bright enough object, the error on the PSF estimated from the AO loop data is dominated by the turbulence noise (Veran 1997). Therefore, we have:

$P S D_{\mathrm{h}}(\boldsymbol{f})=\sigma_{\text {turb }}^{2}(\boldsymbol{f})$.

However, if some other errors are introduced in the calculation of $h_{\mathrm{m}}$ (unexpected fixed aberrations for example), $P S D_{\mathrm{h}}(\boldsymbol{f})$ should be increased accordingly.

\section{Simulation method and results}

In this section, the myopic deconvolution performance is studied. In order to show the influence of the turbulence noise on the deconvolution process and the gain brought by a myopic regularization, a very partial $\mathrm{AO}$ correction has been simulated as following.

We have considered an AO system operating on a $D=3 \mathrm{~m}$ telescope with a $1 \mathrm{~m}$ central obstruction diameter. The turbulence is characterized by a single layer with a Fried parameter equal to $10 \mathrm{~cm}$ at the imaging wavelength $\lambda=0.5 \mu \mathrm{m}$, a $\bar{v}=20 \mathrm{~m} / \mathrm{s}$ wind velocity. 
The AO system is made up of a $9 \times 9$ Shack-Hartmann wavefront sensor, and a 88 piezo-stack mirror. The servoloop bandwidth is $80 \mathrm{~Hz}$. The simulation tool used was developed at ONERA (Rousset et al. 1991). 1024 instantaneous phase screens are simulated. Each wavefront gives a short exposure PSF. The sample frequency $(500 \mathrm{hz})$ leads to a total integration time of 2 seconds. With such a short integration time, the turbulence noise is not negligeable. In these conditions, and for a 11.5 magnitude object (corresponding to 55 detected photons per sub-aperture and per frame), we obtain a very partial correction characterized by its PSF with a Strehl ratio of 0.03 (ratio between the intensity at the center of the corrected PSF and the intensity of the diffraction limited PSF).

$\tilde{h}_{\mathrm{m}}$ is directly deduced from the simulated wavefronts using Eq. (6). Note that, in this case, we have an ideal measurement of the residual wavefront and therefore an ideal estimation of $\tilde{h}_{\mathrm{m}}$.

The STF is computed by the average of the 1024 square moduli of the short exposure OTFs. The PSD is therefore deduced from Eq. (7).

The corrected image of a binary star, including photon noise, is shown in Fig. $1\left(10^{6}\right.$ detected photons in the whole image). We consider a $128 \times 128$ image sampled at the Nyquist frequency, i.e. with a pixel angular size $\frac{\lambda}{2 D}=0.033 \operatorname{arcsec}($ field of view $=4.2 \operatorname{arcsec}$ ).

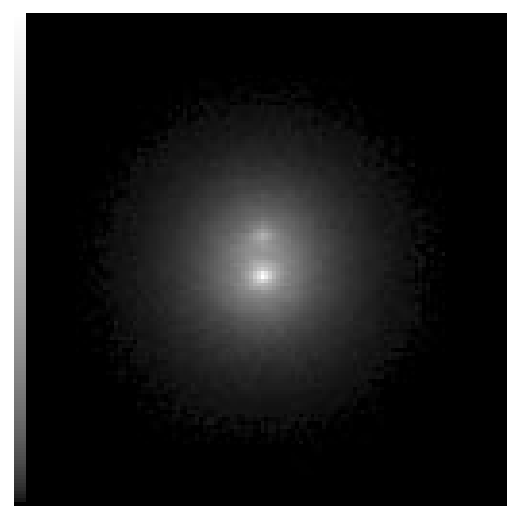

Fig. 1. AO corrected image of a binary star (simulation with Strehl ratio equal to 0.03 ). Total flux $10^{6}$ photons, only photon noise in the image

The separation of the two components is $\Delta$ sep $=10.4$ pixels, i.e., an angular separation of 0.34 arcsec. The intensity of two components are $7.15310^{5}$ and $2.84710^{5}$ photons i.e., a magnitude difference equal to $\Delta m=1$.

The image presented in Fig. 1 clearly shows the need of a deconvolution process for the estimation of the star positions and magnitudes.

A pixel-by-pixel deconvolution (no reparametrization of the object) gives good results (Conan et al. 1997; Mugnier et al. 1998), but the star positions are known only within a pixel and the photometry measurement is not very accurate because of the residual flux in the background after deconvolution. The multiple-star object model avoids both of these limitations. Note, however, that the number of stars is assumed to be known. In practice, this number may be difficult to estimate from the long exposure image, in particular in low Signal to Noise Ratio (SNR) conditions. In this case, it is possible to perform a two step deconvolution process: a pixel-by-pixel deconvolution to estimate the number of star and a "multiple-star object model" deconvolution to improve the astrometry and the photometry. The ultimate performance, in case of very low flux SNR, are currently being investigated. A more sophisticated scheme would involve the simultaneous detection of the number of stars and the estimation of their parameters (Champagnat et al. 1996).

Comparison beetwen pixel-by-pixel and reparametric deconvolution are presented in (Mugnier et al. 1998). In the following, one will focus on the reparametric deconvolution and especially the gain brought by a myopic deconvolution.

Table 1 shows the statistics of the deconvolved parameters over 50 different photon noise realizations. The results are obtained by processing the image with the following techniques:

- a crude estimation (value and position of the two maxima in the image)

- a classical deconvolution. The first term of the criterion defined in Eq. (5) is minimized assuming that PSF is constant and equal to $h_{\mathrm{m}}$.

- a blind deconvolution. The sole first term of the criterion defined in Eq. (5) is minimized with respect to the object parameters and PSF (i.e., no PSF regularization)

- a myopic deconvolution, as presented above (the sum of all terms of Eq. (5) are minimized).

It shows that a deconvolution and especially a myopic deconvolution method increases the accuracy of the parameter estimation.

The classical deconvolution gives a very good result on the star separation: a sub-pixel precision, no bias and an accuracy (standard deviation) about 66 times lower than the diffraction limit $\left(\frac{\lambda}{D}\right)$. Nevertheless, the problem of the classical deconvolution lies in the deficiency of the flux estimation $(\simeq 7 \%)$. This error is mostly due to a bad restitution of the global flux $\left(\alpha_{1}+\alpha_{2}\right)$ which is a free parameter in the deconvolution process. This evolution may be important to take into account the possible loss of flux in the image, due to stars bordering the field of view.

The introduction of the myopic deconvolution leads to better photometric results (a better restitution of the global flux). The error on $\alpha_{1 \text { es }}$ and $\alpha_{2 \text { es }}$ is significantly reduced $(2.64 \%)$.

The separation estimation, which is already good for the classical case is not improved by the myopic one. 
Table 1. Simulation results: $\left\langle\Delta\right.$ sep $>$ and $\sigma_{\Delta \text { sep }}$ are the average and the standard deviation of the separation (pixel unit and arcsecond unit) between the two components calculated on 50 different noise realizations. $\langle\Delta I\rangle$ and $\sigma_{I}$ are the average and the standard deviation of the global error between the true and estimated intensities of the two components in photon unit. They are calculated on 50 different noise realizations $\left(\Delta I=100 \sqrt{\left|\alpha_{1}-\alpha_{1 \mathrm{es}}\right|^{2}+\left|\alpha_{2}-\alpha_{2 \mathrm{es}}\right|^{2}} /\left(\alpha_{1}+\alpha_{2}\right)\right)$. Recall that 1 pix $=\frac{\lambda}{2 D}=0.033$ arcsecond

\begin{tabular}{|c||c|c||c|c|}
\hline & $\begin{array}{c}<\Delta \text { sep } \\
(\mathrm{pix})\end{array}$ & $\begin{array}{c}\sigma_{\Delta \text { sep }} \\
(\mathrm{pix})\end{array}$ & $\begin{array}{c}\langle \% I \\
(\%)\end{array}$ & $\begin{array}{c}\sigma_{I} \\
(\%)\end{array}$ \\
\hline $\begin{array}{c}\text { crude } \\
\text { estimation }\end{array}$ & 10.66 & 0.47 & 76.64 & 6.64 \\
\hline $\begin{array}{c}\text { classical } \\
\text { deconvolution }\end{array}$ & 10.40 & 0.03 & 6.69 & 0.16 \\
\hline $\begin{array}{c}\text { blind } \\
\text { deconvolution }\end{array}$ & 10.41 & 0.23 & 2.95 & 0.96 \\
\hline $\begin{array}{c}\text { myopic } \\
\text { Deconvolution }\end{array}$ & 10.40 & 0.04 & 2.64 & 0.32 \\
\hline $\begin{array}{c}\text { True object } \\
\text { parameters }\end{array}$ & 10.40 & & & \\
\hline
\end{tabular}

The accuracy is even slightly lower than in the classical case (about $\frac{\lambda}{50 D}$ ) but it is not significant. The good estimation, obtained here in the classical case, is most likely due to the fact that the mean PSF is a good estimate of the simulated PSF (circular symmetry, no static aberrations, only turbulent noise) and is good enough for the estimation of the separation. Yet, the accuracy of the star positions should be increased by the myopic deconvolution in the case of a badly estimated PSF.

The blind deconvolution, which is not regularized, is markedly less stable than the myopic one, in the sense that estimated object parameters are more dependent on the noise outcomes on the image (important increase of the standard deviations) and are consequently less accurate.

In short, the classical deconvolution is good enough for an astrometric estimation, but the myopic deconvolution is needed to improve the object photometry (by a factor 3.5 in our case). As described below, it also leads to a better PSF estimation. A good PSF determination is important because the myopic deconvolution is a joint estimation on the object and the PSF. Therefore, an accurate PSF restitution leads to an accurate estimation of the object parameters.

Figure 2 shows a cut of the different normalized OTF. It shows that a blind or a myopic deconvolution improves the accuracy of the OTF at low frequencies (where the signal to noise ratio is high). But the blind deconvolution is limited by the photon noise. The noise has been propagated, and amplified, from the image to the estimated PSF (the expected behavior in a non-regularized case).

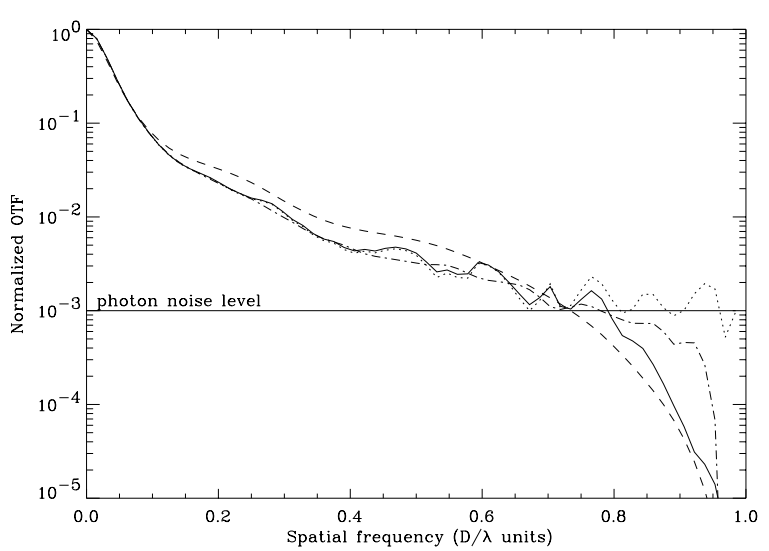

Fig. 2. Cut off normalized Optical Transfer Function versus spatial frequency: true AO corrected OTF (dashed-dotted line), mean OTF (dashed line), Blind deconvolved OTF (dotted line) and Myopic deconvolved OTF (solid line) are shown for comparison. The spatial frequency is normalized to the telescope cutoff frequency. The photon noise level $\frac{1}{N}$ is indicated for comparison

On the other hand, the myopic deconvolution allows a better restitution of the PSF even at high frequencies, and avoid the noise propagation and amplification.

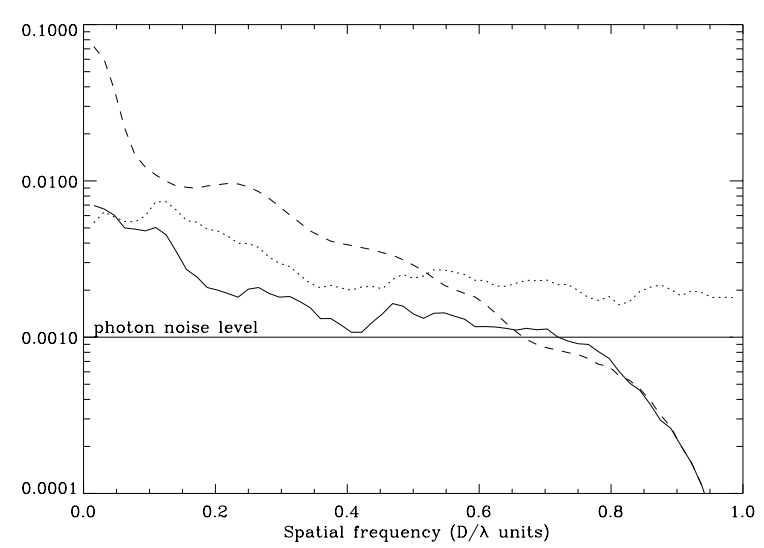

Fig. 3. Circular average of the modulus of the difference between: true OTF and mean OTF (dashed line), true OTF and Blind deconvolved OTF (dotted line), true OTF and Myopic deconvolved OTF (solid line). The photon noise level $\frac{1}{N}$ is indicated for comparison

Figure 3 allows a frequency to frequency assessment of the gain brought by the myopic deconvolution on the PSF. The influence of the regularization is visible at high frequencies, where the myopic deconvolved OTF follows the mean OTF and decreases below the photon noise level.

The average distance between the true PSF and its different estimations, $<\sqrt{\left|P S F_{\text {true }}-P S F_{\text {est }}\right|^{2}}>$, is a 
good summary of the Figs. 2 and 3 and quantifies the gain brought by the myopic deconvolution on the PSF:

- distance between the true and the mean PSF: $2.7210^{-5}$

- average and standard deviation of the distance between the true and the blind deconvolved PSF: $2.22 \pm$ $0.0810^{-5}$

- average and standard deviation of the distance between the true and the myopic deconvolved PSF: $1.05 \pm 0.0310^{-5}$.

The values are given for a unit energy PSF.

The simulations have shown that, in the case of very partial AO correction (ie a strong turbulent noise), a myopic deconvolution increases the accuracy of the joint estimation on the object parameters and the PSF. It leads, in particular, to good photometry estimation.

\section{Experimental results}

We have applied our myopic deconvolution method to real images acquired with PUEO, the Canada-France-Hawaii Telescope adaptive optics system operated at the top of Mauna Kea in Hawaii. PUEO is a low order (19 actuators) curvature based system. Figure 4 shows the image of binary star HD 203024 in $H$ band $(1.65 \mu \mathrm{m})$. This image was acquired in June 1996 using the $256 \times 256$ Monica near infrared camera provided by University of Montreal. The plate scale is 0.034 arcsec/pixel and the integration time was 3 minutes. The object was bright enough $\left(m_{R}=9.5\right)$ to be also used as the reference for AO guiding and the WFS could be used at its nominal $1 \mathrm{kHz}$ sampling frequency. During the acquisition, the estimated seeing was $0.76 \operatorname{arcsec}\left(r_{0}=13.5 \mathrm{~cm}\right.$ at $\left.\lambda=0.5 \mu \mathrm{m}\right)$. At the top of Mauna Kea, the wind speed is typically $\bar{v}=20 \mathrm{~m} / \mathrm{s}$. A flat field and a background are subtract from the image. The "bad" pixels (cosmic ray events, aberrant pixels of the camera...) are replaced by a mean of the neighbouring pixels before deconvolution.

The PSF associated with the image was retrieved from the loop data using the method described in (Veran et al. 1997). The estimated Strehl ratio is $33 \%$. For an object of this magnitude, the precision of the estimated PSF is normally limited by the turbulence noise. The PSF estimated from the loop data should be accurate enough for a classical deconvolution. In this particular case however, uncalibrated static aberrations degrade the reconstruction and a myopic deconvolution is necessary.

Using the reconstructed PSF and a classical pixel to pixel deconvolution algorithm such as Lucy-Richardson, the deconvolved object presents two sources which seem to have a faint companion at the very same relative position. This is bound to be an artifact. This artifact can be traced

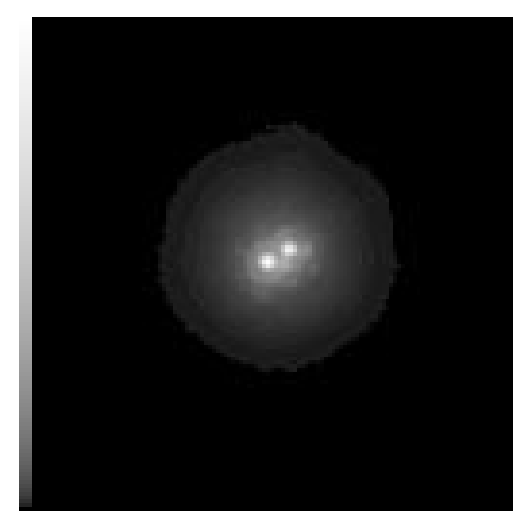

Fig. 4. AO corrected image of binary star HD 203024 in $H$ band $(\mathrm{SR} \simeq 33 \%) .128 \times 128$ pixels image corresponding to a 4.35 arcsec field of view

to the presence of a bump on the first Airy ring of the real PSF which does not appear in the reconstructed PSF.

The classical deconvolution (i.e, the deconvolution method using the object reparametrization but assuming that the mean PSF - estimated with WFS data - is the real one) allows, of course, the restoration of a binary star (object reparametrization). The problem is that the PSF artifacts are not taken into account. It leads to errors on the estimation of the parameters, especially on the flux.

As shown in Fig. 5, the real PSF can nevertheless be retrieved using our myopic deconvolution algorithm, in spite of the fact that Eq. (9) under-estimates $P S D_{\mathrm{h}}$ (the static aberrations are not taken into account in the PSD, because they are not seen by the WFS). After myopic deconvolution, a bump clearly appears at the first Airy ring in the the estimated PSF. We have generally noticed that with our stochastic approach no hyper-parameter is needed. Yet, in this particular case of an under-estimated PSD, one could add an ad-hoc hyper-parameter smaller then 1 on the PSF regularization term in Eq. (5) to loosen the constraint.

An even better accuracy might be obtained, if we were able to quantify the static aberration effects in the Fourier domain in order to add them in the regularization term.

In Table 2, we compare the results of the classical and the myopic deconvolution method. If the estimated star separation is nearly the same in both cases, a significant difference is found in the estimated flux. According to the simulation results, the myopic deconvolution improves the global flux, and therefore the accuracy of the magnitude restoration of each star. It would be useful to have a photometric calibration star to obtain the atmospheric coefficient of absorption and to give the real magnitude of each star; unfortunately no such data are available for this observating night.

In short, the myopic deconvolution allows to take into account unpredictable phenomenons (turbulence noise, 


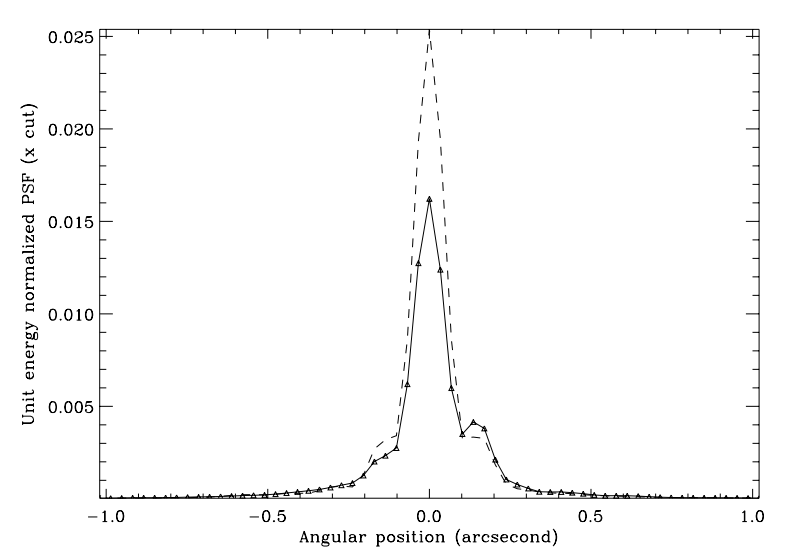

Fig. 5. Comparison of the WFS reconstructed mean PSF (dotted line) and the myopic estimated PSF (solid line). Normalized square root of $x$-axis cut are represented

Table 2. Experimental results: $\Delta$ sep is the separation between the two components, pixel unit (first column) and arcsecond units (second column). $\Delta m$ the magnitude difference. Last column gives the total flux of the two components (in detected photons). In comparison, the total flux in the image is $4.8610^{8}$ detected photons

\begin{tabular}{|c||c|c||c|c|}
\hline & $\begin{array}{c}\Delta \text { sep } \\
\text { (pixel) }\end{array}$ & $\begin{array}{c}\Delta \text { sep } \\
\text { (as) }\end{array}$ & $\Delta m$ & total flux \\
\hline classical deconvolution & 6.58 & 0.224 & 0.10 & $3.9710^{8}$ \\
\hline myopic deconvolution & 6.58 & 0.224 & 0.13 & $4.8610^{8}$ \\
\hline
\end{tabular}

uncalibrated static aberrations...) for a better accuracy of the object photometry.

\section{Conclusions}

The AO correction of an image theorically allows to reach the diffraction limit of ground based telescope. Unfortunately this correction is always partial and the image is still blurred by the residual PSF.

In this article, we have presented a myopic deconvolution method for stellar fields based on the a priori knowledge we have of the object and the PSF. The object knowledge leads us to reparametrize our object model. The structure and variability of the PSF given by the WFS measurements are introduced as first (mean) and second (PSD) order moments in an a priori probability law.

We have shown on simulations that this technique allows a good restoration of the object (position and photometry) and of the PSF. Experimental data have been also successfully processed.

Future work should implement other probability statistics. Non-stationary Gaussian or Poisson plus Gaussian statistics should be used in order to provide a better noise model.

A current limitation of our method is that it does not include a systematic detection of stars. Up to now, the number of star is first estimated on the image then the residual of the deconvolution is checked for missed stars. A Bernouilli-Gaussian or P-Gaussian statistic should permit the inclusion of an automatic star detection in the algorithm.

Also, the estimation of a theorical error calculation on the star parameters should be added to better quantify the precision of the estimations.

Lastly, in the case of a field larger than the isoplanatic path, a space variant PSF could be introduced to take into account anisoplanatic effects. The advantage of our multiple-star object model in addition with a myopic approach is that one can easily incorporate one PSF for each star as additional unkowns. In that case, another regularization term should be added to account for the angular correlation of the PSF in the field.

Acknowledgements. The authors wish to thank V. Michau and G. Rousset for fruitful discussions and helpful comments.

\section{References}

Babcock H.W., 1953, PASP 65, 229

Champagnat F., Goussard Y., Idier J., 1996, IEEE S.P. 44, 12, 2988

Christou J.C., Bonaccini D., Ageorges N., 1997, in: Adaptive Optics and Applications, Vol. 3126, SPIE, pp. 68-80

Conan J.-M., 1994, Ph. D. thesis, Université Paris XI Orsay

Conan J.-M., Mugnier L., Fusco T., Michau V., Rousset G., 1997, in: Adaptive Optics and Applications, Vol. 3126, SPIE, pp. 56-67

Conan J.-M., Mugnier L., Fusco T., Michau V., Rousset G., 1998, Appl. Opt. 37, 21, pp. 4614-4622

Conan J.-M., Rousset G., Madec P.-Y., 1995, J. Opt. Soc. Am. A $12,7,1559$

Demoment G., 1989, IEEE Trans. Acoust. Speech Signal Process. 37, 12, 2024

Fried D.L., 1965, J. Opt. Soc. Am. 55, 11, 1427

Gunsay M., Jeffs D., 1995, in: IEEE Trans. Image Proc., Vol. 4, IEEE, pp. 1602-1612

Hogbom J., 1974, A\&A 15, 417

Hook R., Lucy L., 1994, in: Hanish R., White R. (eds.), The Restoration of HST Image and Spectra II, pp. 86-92

Knox K.T., Thompson B.J., 1974, Astrophys. J. Lett. 193, L45

Labeyrie A., 1970, A\&A 6, 85

Lucy L., 1994, in: Hanish R., White R. (eds.), The Restoration of HST Image and Spectra II, pp. 79-85

Mugnier L.M., Conan J.-M., Fusco T., Michau V., 1998, in: Bayesian Inference for Inverse problems, SPIE, San Diego, CA, U.S.A.

Northcott M., 1994, in: Ealey M.A., Merkle F. (eds.), Adaptive Optics in Astronomy, Vol. 2201, SPIE, pp. 129-136

Rigaut F., Rousset G., Kern P., et al., 1991, A\&A 250, 280

Roddier F., 1981, in: Wolf E. (ed.), Progress in Optics, Vol. XIX, North Holland, Amsterdam, pp. 281-376 
Roddier F., Gilli J.-M., Lund G., 1982, J. Opt. (Paris) 13, 263

Rousset G., Fontanella J.-C., Kern P., 1990, A\&A 230, 29

Rousset G., Madec P.-Y., Rabaud D., 1991, in: J.M. Beckers and F. Merkle (eds.), High-Resolution Imaging by Interferometry II, No. 39 in ESO Conference and Workshop Proceedings, ESO, ESO, Garching Germany, pp. 10951104
Stetson P., 1987, PASP 99, 465

Thiébaut E., Conan J.-M., 1995, J. Opt. Soc. Am. A 12, 485

Tikhonov A., Arsenin V., 1977, Solutions of Ill-Posed Problems, Winston, DC

Véran J.-P., Rigaut F., Maître H., Rouan D., 1997, J. Opt. Soc. Am. A 14, 3057

Weigelt G., 1977, Opt. Commun. 21, 55 